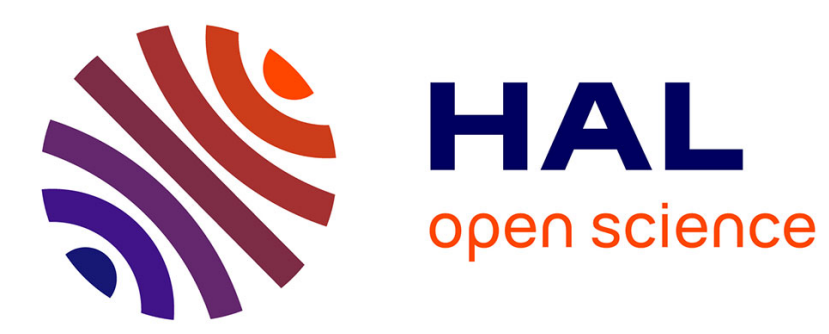

\title{
Statistical analysis of pulse propagation driven by polarization-mode dispersion
}

\author{
Josselin Garnier, Julien Fatome, Gaëlle Le Meur
}

\section{To cite this version:}

Josselin Garnier, Julien Fatome, Gaëlle Le Meur. Statistical analysis of pulse propagation driven by polarization-mode dispersion. Journal of the Optical Society of America B, 2002, 19 (9), pp.1968-1977. hal-00475127

\section{HAL Id: hal-00475127 \\ https://hal.science/hal-00475127}

Submitted on 21 Apr 2010

HAL is a multi-disciplinary open access archive for the deposit and dissemination of scientific research documents, whether they are published or not. The documents may come from teaching and research institutions in France or abroad, or from public or private research centers.
L'archive ouverte pluridisciplinaire HAL, est destinée au dépôt et à la diffusion de documents scientifiques de niveau recherche, publiés ou non, émanant des établissements d'enseignement et de recherche français ou étrangers, des laboratoires publics ou privés. 


\title{
Statistical analysis of pulse propagation driven by polarization mode dispersion
}

\author{
J. Garnier \\ Laboratoire de Statistique et Probabilités, \\ Université Paul Sabatier, 118 Route de Narbonne, 31062 Toulouse Cedex 4, France, \\ Tel. (33) 5615562 20, Fax. (33) 5615560 89, Email: garnier@cict.fr \\ J. Fatome * and G. Le Meur \\ Alcatel Research and Innovation, \\ Route de Nozay, 91460 Marcoussis, France \\ (Preliminary version - January 29, 2002)
}

\begin{abstract}
This paper considers the propagation of linear pulses driven by random polarization mode dispersion. Analytical expressions are derived for the probability density functions of the pulse width, timing displacement, and degree of polarization. The study is performed in Stokes space, and the frequency correlation between modes is shown to play an important role.
\end{abstract}

\section{INTRODUCTION}

The study of pulse propagation in a birefringent fiber with random birefringence has become of great interest for telecommunication applications. Recent experiments have shown that Polarization Mode Dispersion (PMD) is one of the main limitations on fiber transmission links [1]. PMD occurs because single-mode fibers are not really single-mode but can exhibit different group velocities. PMD is thus characterized by a Differential Group Delay (DGD) between the two modes associated with the fiber. PMD results in the splitting of a polarized input pulse into two orthogonal polarizations that arrive at the ouput at different times.

The effects of PMD are usually treated by means of the three-dimensional PMD vector. The PMD vector $\hat{\mathbf{r}}$ gives in Stokes space the relation between the output state and the frequency derivative of the output state: $\hat{\mathbf{s}}^{\prime}(\omega)=\hat{\mathbf{r}}(\omega) \times \hat{\mathbf{s}}(\omega)$. The Principal States of Polarization (PSP) are defined as the states that satisfy $\hat{\mathbf{r}}(\omega) \times \hat{\mathbf{s}}(\omega)=\mathbf{0}$, so that no change in output polarization can be observed close to these states at first order in $\omega$. However, for broadband pulses the first-order approach is not valid anymore, the changes of the PSP and the length of $\hat{\mathbf{r}}(\omega)$, i.e. the DGD, with respect to frequency cannot be neglected. As a consequence the frequency dependences of the PMD vectors and DGD are worth studying.

For a given frequency the properties of the PMD vector are well known [2-4]. In particular its elements are independent Gaussian random variables. Despite its obvious relevance the frequency dependences of the PMD vectors and DGD have been treated only in a few papers $[5,6]$. Furthermore each paper deals with the computation of one particular expectation: the expectation of the correlation between two PMD vectors [5], the expectation of the correlation between two square DGD [6]. We shall be able to extend these results by using an approach based on the theory on stochastic differential equations. This theory will give us the means to compute any expectation of any combination of PMD vectors or DGD at different frequencies. Furthermore, by applying and extending the theory of moments [7] we shall deduce from these formulas closed form expression for the means, variances and probability density functions of relevant quantities for the characterization of pulse propagation such as the time displacement, the time rms width, and the degree of polarization.

\section{DESCRIPTION OF THE MODEL}

The evolution of polarized fields in randomly birefringent fibers is governed by the coupled Schrödinger equations with random PMD between two modes (polarizations) [8]:

$$
i \mathbf{A}_{z}+K_{0} \mathbf{A}+i K_{1} \mathbf{A}_{t}-\frac{\beta^{\prime \prime}}{2} \mathbf{A}_{t t}=0
$$

where $\mathbf{A}$ is the row vector $\left(A_{x}, A_{y}\right)^{T}$ that denotes the envelopes of the electric field in the two eigenmodes. The matrices $K_{0}$ and $K_{1}$ describe random fiber birefringence. The Group Velocity Dispersion (GVD) coefficient is the second derivative of the propagation constant with respect to frequency $\beta^{\prime \prime}$, which is positive (resp. negative) for normal (resp. anomalous) dispersion. One can eliminate the fast random birefringence variations that appear in Eq. (1) by means of a change of variables, that leads to the new vector equation:

$$
i \mathbf{U}_{z}-\frac{\beta^{\prime \prime}}{2} \mathbf{U}_{t t}=i R \mathbf{U}_{t}
$$

where $\mathbf{U} \equiv M^{-1} \mathbf{A}, \mathbf{U}=(u, v)^{T}$ represents the slow evolution of the field envelopes in the reference frame of the local polarization eigenmodes, and the matrix $M$ obeys the equation $i M_{z}+K_{0} M=0 . R$ is a $z$-dependent matrix that involves high order PMD. It is associated with the coupling between the modes, as well as an accumulation of a mismatch between their phases.

The most commonly used model is the so-called retarded-plate model $[9,10]$. The birefringence strength 
$\Delta \beta$ and its derivative $\Delta \beta^{\prime}$ are constant; the birefringence angle is constant over elementary intervals with length $\Delta z$; at junctions between the fiber pieces with length $\Delta z$, a random axial rotation is added as well as an addition of random phase difference between the two field components, so that the Stokes vector obeys a random walk over the Poincaré sphere. For our numerical simulations we shall use this model. If $\Delta z$ is small enough we can model this configuration by considering that the matrix $R$ is [10]:

$$
R(z)=m_{1}(z) \Sigma_{1}+m_{2}(z) \Sigma_{2}+m_{3}(z) \Sigma_{3},
$$

where $\Sigma_{j}$ are Pauli matrices:

$\Sigma_{1}=\left(\begin{array}{cc}0 & 1 \\ 1 & 0\end{array}\right), \quad \Sigma_{2}=\left(\begin{array}{cc}0 & -i \\ i & 0\end{array}\right), \quad \Sigma_{3}=\left(\begin{array}{cc}1 & 0 \\ 0 & -1\end{array}\right)$,

and the real-valued processes $m_{j}$ are random white noises with autocorrelation functions

$$
\left\langle m_{j}(z) m_{j}\left(z^{\prime}\right)\right\rangle=\sigma^{2} \delta\left(z-z^{\prime}\right) \text { with } \sigma^{2}=\frac{\Delta \beta^{\prime 2} \Delta z}{12} .
$$

In this model the so-called PMD parameter is given by $D_{p}=\sqrt{8 /(3 \pi)} \Delta \beta^{\prime} \sqrt{\Delta z}[10]$. Our parameter $\sigma^{2}$ is simply related to $D_{p}$ through:

$$
\sigma^{2}=\frac{\pi}{32} D_{p}^{2}
$$

All models of random birefringence are eventually described by the white noise model (3) with some effective parameter $\sigma$ (or $D_{p}$ ) as soon as the correlation length of the random fluctuations of birefringence parameters is much smaller than the other characteristic lengths of the problem $[4,13,14]$.

For consistency, note that the usual GVD parameter is $D=-2 \pi c \beta^{\prime \prime} / \lambda^{2}$ where $\lambda$ is the carrier wavelength of the pulse $(1.55 \mu \mathrm{m}$ for standard optical fiber applications). Typical values of the PMD parameter $D_{p}$ have been measured in the range $0.1-1 \mathrm{ps} / \sqrt{\mathrm{km}}[11,12]$. Dispersion shifted fibers (which are of particular interest for telecommunication) have been found to have particular high values [12]. The correlation length $\Delta z$ of PMD varies around $0.1-1 \mathrm{~km}$, and the GVD parameter $D$ is between $1-20 \mathrm{ps} / \mathrm{nm} / \mathrm{km}$.

\section{PULSE CHARACTERIZATION}

Numerical simulations have been carried out for the determination of the width and degree of polarization of the pulse versus the propagation distance $[9,15]$. In these simulations the effect of GVD was usually neglected to examine the effect of PMD exclusively. Empirical formulas for the pulse width and degree polarization were proposed. Furthermore the numbers of samples necessary to get stable averaged values were found to be very large, which tends to prove that the variances of the pulse width and degree of polarization are large as well. We shall confirm theoretically these observations. In this section we shall derive closed-form expressions for the average values, variances, and Probability Density Functions (PDF) of the time displacement, pulse width, and degree of polarization of a pulse driven by randomly varying birefringence. We add the time displacement because the fiber PMD not only induces optical pulse broadening but also differences in pulse arrival times between different fiber realizations.

If we neglect GVD then the driving equation for the field is:

$$
\mathbf{U}_{z}=R(z) \mathbf{U}_{t}
$$

where the $z$-dependent matrix $R$ is the random combination (3) of the Pauli matrices. We now define the parameters of the pulse that we shall study in the following sections.

Time displacement. We first introduce the time displacement $T_{c}$ :

$$
T_{c}=\frac{\int t\left(|u|^{2}+|v|^{2}\right) d t}{\int\left(|u|^{2}+|v|^{2}\right) d t}
$$

Pulse polarization. If the initial pulse is polarized along the $u$-axis, then the rotation of the pulse polarization can be characterized by the parameter $P_{r}$ defined as the ratio of the energy on the $u$-axis over the total energy:

$$
P_{r}=\frac{\int|u|^{2} d t}{\int\left(|u|^{2}+|v|^{2}\right) d t}
$$

The degree of polarization is defined by [17]:

$$
P_{d}=\frac{\sqrt{s_{1}^{2}+s_{2}^{2}+s_{3}^{3}}}{s_{0}}
$$

in terms of the Stokes parameters:

$$
\begin{aligned}
& s_{0}=\int\left(|u|^{2}+|v|^{2}\right)(t) d t, \\
& s_{1}=\int\left(|u|^{2}-|v|^{2}\right)(t) d t, \\
& s_{2}=2 \int \operatorname{Re}\left(u^{*} v\right)(t) d t, \\
& s_{3}=2 \int \operatorname{Im}\left(u^{*} v\right)(t) d t .
\end{aligned}
$$

We can also introduce the degree of polarization by the following way. We construct the new field components $\left(u_{\theta, \xi}, v_{\theta, \xi}\right)$ as

$$
\begin{aligned}
& u_{\theta, \xi}(t)=\cos (\theta) u(t)+\sin (\theta) e^{i \xi} v(t) \\
& v_{\theta, \xi}(t)=-\sin (\theta) u(t)+\cos (\theta) e^{i \xi} v(t)
\end{aligned}
$$


where $\theta$ and $\xi$ are determined so that the energy in the component $u_{\theta, \xi}$ is maximized. The angle $\theta$ is the socalled polarization angle while the ratio of the energy of the component $u_{\theta, \xi}$ over the total energy:

$$
P_{d 1}=\frac{\int\left|u_{\theta, \xi}\right|^{2} d t}{\int\left(|u|^{2}+|v|^{2}\right) d t}
$$

is related to the degree of polarization through the simple identity $P_{d}=2 P_{d 1}-1$. Note that $P_{d 1} \geq P_{r}$. Furthermore the degree of polarization of a monochromatic pulse is always equal to 1 , whatever the rotation of polarization. However, the spectral components of a short pulse tend to loose their polarization coherence so that the degree of polarization $P_{d}$ of the corresponding pulse decays to zero.

Pulse width. It is not clear for practical applications whether the timing displacement should be eliminated from the computation of the pulse broadening. If the speed of the timing displacement is so slow that the clock recovery can follow the change, then the timing displacement has no influence on system performance and should be eliminated. Accordingly the pulse width should be defined by:

$$
T_{w 1}^{2}=\frac{\int\left(t-T_{c}\right)^{2}\left(|u|^{2}+|v|^{2}\right) d t}{\int\left(|u|^{2}+|v|^{2}\right) d t}
$$

If the PMD fluctuates more rapidly than the clock recovery can track, then it is necessary to include the timing displacement to properly simulate the transmission system:

$$
T_{w 2}^{2}=\frac{\int t^{2}\left(|u|^{2}+|v|^{2}\right) d t}{\int\left(|u|^{2}+|v|^{2}\right) d t}=T_{w 1}^{2}+T_{c}^{2}
$$

We shall compute the mean values, variances, and PDF of these quantities for a general initial pulse $u_{0}$ polarized along the $u$-axis with $\mathrm{rms}$ width $T_{0}$. We shall also give explicit formulas in the case of a Gaussian pulse with rms width $T_{0}$ :

$$
u_{0}(t)=\exp \left(-\frac{t^{2}}{4 T_{0}^{2}}\right)
$$

We shall also consider a sech pulse with rms width $T_{0}$ whose expression is:

$$
u_{0}(t)=\operatorname{sech}\left(\frac{\pi t}{2 \sqrt{3} T_{0}}\right)
$$

\section{THE STOKES VECTOR}

The Fourier components $\hat{\mathbf{U}}:=(\hat{u}, \hat{v})^{T}$ of the field:

$$
\hat{u}(\omega)=\int u(t) \exp (i \omega t) d t, \quad \hat{v}(\omega)=\int v(t) \exp (i \omega t) d t
$$

obey a system of ordinary differential equations:

$$
\hat{\mathbf{U}}_{z}=i \omega R(z) \hat{\mathbf{U}}
$$

There exist simple and exact analytical identities between the amount of broadening and Fourier components $[7,16]$, as well as between the polarization degree, the time displacement and the Fourier components. These formulas are in fact nothing more than standard Parseval formula applied to well chosen quantities. A convenient representation of the polarization evolutions induced by the fluctuations $m_{j}$ may be done in terms of the Stokes vector $\hat{\mathbf{s}}$ associated to the Fourier components of the field $(u, v)$ :

$$
\begin{aligned}
& \hat{s}_{1}(\omega)=\left(|\hat{u}|^{2}-|\hat{v}|^{2}\right)(\omega), \\
& \hat{s}_{2}(\omega)=2 \operatorname{Re}\left(\hat{u}^{*} \hat{v}\right)(\omega), \\
& \hat{s}_{3}(\omega)=2 \operatorname{Im}\left(\hat{u}^{*} \hat{v}\right)(\omega),
\end{aligned}
$$

whose modulus $\sqrt{\hat{s}_{1}^{2}+\hat{s}_{2}^{2}+\hat{s}_{3}^{2}}=|\hat{u}|^{2}+|\hat{v}|^{2}=\hat{E}_{0}(\omega)$ is the spectral intensity:

$$
\hat{E}_{0}(\omega)=\left|\int u_{0}(t) e^{i \omega t} d t\right|^{2}
$$

which is a preserved quantity. In terms of the Stokes parameters the dynamics induced by PMD is simple:

$$
\hat{\mathbf{s}}_{z}=2 \sigma \omega \dot{\mathbf{W}}(z) \times \hat{\mathbf{s}}
$$

where $\sigma \dot{\mathbf{W}}(z)$ is the row vector $\left(m_{3}, m_{1}, m_{2}\right)^{T}(z)$. Thus the $m_{j}$ appear as elementary infinitesimal generators of random rotations of the Stokes vector over the Poincaré sphere. Mathematically speaking the above equation should be understood as:

$$
\begin{aligned}
& d \hat{s}_{1}(\omega)=2 \sigma \omega\left(\hat{s}_{2} \circ d W^{3}-\hat{s}_{3} \circ d W^{2}\right) \\
& d \hat{s}_{2}(\omega)=2 \sigma \omega\left(\hat{s}_{3} \circ d W^{1}-\hat{s}_{1} \circ d W^{3}\right) \\
& d \hat{s}_{3}(\omega)=2 \sigma \omega\left(\hat{s}_{1} \circ d W^{2}-\hat{s}_{2} \circ d W^{1}\right)
\end{aligned}
$$

where $\circ$ stands for the Stratonovich stochastic integral and $W^{j}$ are three independent Brownian motions. The correlation degree between the Stokes vectors at two nearby frequencies $\omega_{1}$ and $\omega_{2}$ is:

$$
C\left(\omega_{1}, \omega_{2}\right):=\frac{\hat{\mathbf{s}}\left(\omega_{1}\right) \cdot \hat{\mathbf{s}}\left(\omega_{2}\right)}{\hat{E}_{0}\left(\omega_{1}\right) \hat{E}_{0}\left(\omega_{2}\right)}
$$

It is easy to check from Eq. (14) that $C$ is a diffusion process with infinitesimal generator:

$$
\mathcal{L}=2 \sigma^{2} \Delta \omega^{2} \frac{\partial}{\partial C}\left(1-C^{2}\right) \frac{\partial}{\partial C}
$$

where $\Delta \omega=\omega_{1}-\omega_{2}$. This means that any expectation of a smooth function $f(C)$ of $C$ satisfies the forward Kolmogorov equation:

$$
\frac{\partial\langle f(C)\rangle}{\partial z}=\langle\mathcal{L} f(C)\rangle
$$


In particular the mean value of $C$ decays exponentially as:

$$
\left\langle C\left(\omega_{1}, \omega_{2}\right)\right\rangle=\exp \left(-4 \Delta \omega^{2} \sigma^{2} z\right)
$$

This shows that PMD is a strongly frequency-dependent phenomenon, and that the Stokes vectors at two nearby frequencies become statistically independent as $z$ increases. More precisely we can derive from the expression of the infinitesimal generator the Fokker-Planck equation for the PDF $p(z, C)$ satisfied by the correlation degree $C\left(\omega_{1}, \omega_{2}\right)$ :

$$
\frac{\partial p}{\partial z}=2 \sigma^{2} \Delta \omega^{2}\left(\left(1-C^{2}\right) \frac{\partial^{2} p}{\partial C^{2}}-2 C \frac{\partial p}{\partial C}\right)
$$

starting from the initial condition $p(0, C)=\delta(C-1)$. This equation can be solved by means of an expansion over the Legendre polynomials [18]:

$p(z, C)=\frac{1}{2} \sum_{n=0}^{\infty} P_{n}(C) \exp \left(-2 n(n+1) \sigma^{2} \Delta \omega^{2} z\right) \mathbf{1}_{C \in[-1,1]}$

The PDF $p(z,$.$) is plotted in Figure 1$ for different values of $z$. We can thus observe the transition from full correlation $p(0, C)=\delta(C-1)$ at $z=0$ to complete uncorrelation $p(\infty, C)=\frac{1}{2} \mathbf{1}_{C \in[-1,1]}$ for $\sigma^{2} \Delta \omega^{2} z>1$.

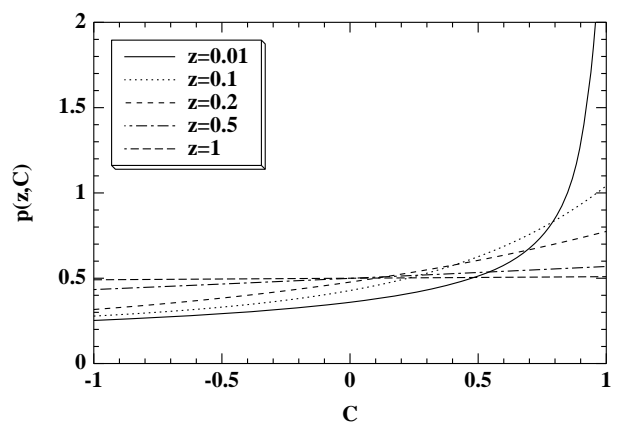

FIG. 1. PDF of the correlation degree $C\left(\omega_{1}, \omega_{2}\right)$ for $\sigma^{2} \Delta \omega^{2}=1$.

\section{TIME DISPLACEMENT}

In terms of the Fourier components the time displacement reads:

$$
T_{c}=\frac{\int \hat{t}(\omega) \hat{E}_{0}(\omega) d \omega}{\int \hat{E}_{0}(\omega) d \omega}
$$

where

$$
\hat{t}(\omega):=\frac{\operatorname{Im}\left(\hat{u}^{*}(\omega) \hat{u}^{\prime}(\omega)\right)+\operatorname{Im}\left(\hat{v}^{*}(\omega) \hat{v}^{\prime}(\omega)\right)}{|\hat{u}|^{2}(\omega)+|\hat{v}|^{2}(\omega)},
$$

and the prime stands for the derivative with respect to $\omega$. The process $\bar{t}(\omega, z)$ obeys a simple equation in terms of the Stokes parameters:

$$
d \hat{t}=\sigma\left(\hat{s}_{1} \circ d W^{1}+\hat{s}_{2} \circ d W^{2}+\hat{s}_{3} \circ d W^{3}\right)
$$

Computing the infinitesimal generator of $\hat{t}$ :

$$
\mathcal{L}=\frac{1}{2} \sigma^{2} \frac{\partial^{2}}{\partial \hat{t}^{2}}
$$

we get that $\hat{t}$ obeys the distribution of a Brownian motion with diffusion coefficient $\sigma$. Accordingly the PDF of $\hat{t}(\omega, z)$ is Gaussian:

$$
p(z, \hat{t})=\frac{1}{\sqrt{2 \pi} \sigma \sqrt{z}} \exp \left(-\frac{\hat{t}^{2}}{2 \sigma^{2} z}\right) \mathbf{1}_{\hat{t} \in \mathbb{R}}
$$

For the following results the correlation function of $\hat{t}$ at two nearby frequencies is necessary. It is found that the process $\left(C, \hat{t}_{1}, \hat{t}_{2}\right)$, where $\hat{t}_{1}=\hat{t}\left(\omega_{1}\right), \hat{t}_{2}=\hat{t}\left(\omega_{2}\right)$, and $C=C\left(\omega_{1}, \omega_{2}\right)$, is a diffusion process with infinitesimal generator:

$$
\begin{aligned}
\mathcal{L}= & \frac{1}{2} \sigma^{2} \frac{\partial^{2}}{\partial \hat{t}_{1}^{2}}+\frac{1}{2} \sigma^{2} \frac{\partial^{2}}{\partial \hat{t}_{2}^{2}}+\sigma^{2} C \frac{\partial^{2}}{\partial \hat{t}_{1} \hat{t}_{2}} \\
& +2 \sigma^{2} \Delta \omega^{2} \frac{\partial}{\partial C}\left(1-C^{2}\right) \frac{\partial}{\partial C}
\end{aligned}
$$

We can thus deduce that:

$$
\frac{d}{d z}\left\langle\hat{t}\left(\omega_{1}\right) \hat{t}\left(\omega_{2}\right)\right\rangle=\sigma^{2}\left\langle C\left(\omega_{1}, \omega_{2}\right)\right\rangle
$$

which yields:

$$
\left\langle\hat{t}\left(\omega_{1}\right) \hat{t}\left(\omega_{2}\right)\right\rangle=\frac{1-\exp \left(-4\left(\omega_{1}-\omega_{2}\right)^{2} \sigma^{2} z\right)}{4\left(\omega_{1}-\omega_{2}\right)^{2}}
$$

This allows one to state that the time displacement has mean zero and variance:

$$
\left\langle T_{c}^{2}\right\rangle=\frac{\iint \hat{E}_{0}\left(\omega_{1}\right) \hat{E}_{0}\left(\omega_{2}\right)\left\langle\hat{t}\left(\omega_{1}\right) \hat{t}\left(\omega_{2}\right)\right\rangle d \omega_{1} \omega_{2}}{\left(\int \hat{E}_{0}(\omega) d \omega\right)^{2}}
$$

If the input pulse has Gaussian shape with rms width $T_{0}$, then the variance of the time displacement can be written explicitly:

$$
\left\langle T_{c}^{2}\right\rangle=\frac{T_{0}^{2}}{2}\left(\sqrt{1+\frac{4 \sigma^{2} z}{T_{0}^{2}}}-1\right)
$$

This exact expression depends on the input shape, but the long-term growth rate of the variance is independent of the pulse shape. We can state in great generality that the mean square time displacement first grows linearly for $z \ll T_{0}^{2} / \sigma^{2}$, while the long-range growth is slower $(\sqrt{z})$ :

$$
\begin{aligned}
\left\langle T_{c}^{2}\right\rangle & \stackrel{\sigma^{2} z \ll T_{0}^{2}}{\simeq} \sigma^{2} z-4\left(c_{2}-c_{4}^{2}\right) \frac{\sigma^{4} z^{2}}{T_{0}^{2}} \\
& \stackrel{\sigma^{2} z T_{0}^{2}}{\simeq} \sqrt{\pi} c_{1} \sqrt{\sigma^{2} z}
\end{aligned}
$$

where 


$$
\begin{aligned}
& c_{1}=\frac{\int \hat{E}_{0}(\omega)^{2} d \omega}{\left(\int \hat{E}_{0}(\omega) d \omega\right)^{2}}=\frac{1}{2 \pi} \frac{\int\left|u_{0} \star u_{0}\right|^{2}(t) d t}{\left(\int\left|u_{0}\right|^{2}(t) d t\right)^{2}} \\
& c_{2}=\frac{\int \omega^{2} \hat{E}_{0}(\omega) d \omega}{\int \hat{E}_{0}(\omega) d \omega}=\frac{\int\left|u_{0 t}\right|^{2} d t}{\int\left|u_{0}\right|^{2} d t} \\
& c_{4}=\frac{\int \omega \hat{E}_{0}(\omega) d \omega}{\int \hat{E}_{0}(\omega) d \omega}=\frac{i \int u_{0 t} u_{0}^{*} d t}{\int\left|u_{0}\right|^{2} d t}
\end{aligned}
$$

and the star $\star$ stands for the convolution. An interesting question concerns the statistical distribution of $T_{c}$. We can show on the one hand that the statistical distribution of $T_{c}$ is Gaussian when $\sigma^{2} z \ll T_{0}^{2}$ since $T_{c}$ is then equal to $\sigma W_{z}^{1}$ up to terms of order $\sigma^{2} z / T_{0}^{2}$. On the other hand, when $\sigma^{2} z \gg T_{0}^{2}$ the random variable $T_{c}$ has also Gaussian statistics because Eq. (16) shows that it is the sum of a large number of uncorrelated components. The central limit theorem can then be invoked to show that $T_{c}$ has Gaussian statistics with zero-mean and variance (17). Accordingly we may think that $T_{c}$ has a Gaussian PDF in the general case. However a thorough study shows that the forth moment of $T_{c}$ can be expanded as powers of $\sigma^{2} z / T_{0}^{2}$, and the expansion of $\left\langle T_{c}^{4}\right\rangle$ is different from the corresponding expansion of $3\left\langle T_{c}^{2}\right\rangle^{2}$. This establishes that $T_{c}$ has not strictly Gaussian statistics, although numerical simulations show that the distribution is very close to be Gaussian. This resemblance is not surprising, since the expansion of the high order moments show that the moments of $T_{c}$ obey the standard rules of Gaussian random variables up to order 3 with respect to $\sigma^{2} z / T_{0}^{2}$ :

$$
\left\langle T_{c}^{2 n}\right\rangle=\alpha_{n}\left\langle T_{c}^{2}\right\rangle^{n}\left(1+O\left(\frac{\sigma^{6} z^{3}}{T_{0}^{6}}\right)\right)
$$

where $\alpha_{n}=(2 n+1)(2 n-1) \ldots 3$. In cases of practical applications for which $\sigma^{2} z \leq T_{0}^{2}$, we may thus think at $T_{c}$ as a Gaussian random variable up to a very good approximation.

A second noticeable problem is about the tail of the PDF of $T_{c}$. Although we have just noticed $T_{c}$ has not pure Gaussian statistics, it is the linear combination of Gaussian random variables $\hat{t}$, so that the tail of the distribution of $T_{c}$ will be imposed by the tail of some $\hat{t}(\omega)$. Accordingly:

$$
\mathbb{P}\left(\left|T_{c}\right| \geq \alpha\left\langle T_{c}^{2}\right\rangle^{1 / 2}\right) \stackrel{\alpha \gg 1}{\sim} \frac{1}{\sqrt{2 \pi} \alpha} \exp \left(-\frac{\alpha^{2}}{2}\right)
$$

Note that, if $\sigma^{2} z \leq T_{0}^{2}$ or else if $\sigma^{2} z \gg T_{0}^{2}$ the PDF of $T_{c}$ is exactly:

$$
p(t)=\frac{1}{\sqrt{2 \pi}\left\langle T_{c}^{2}\right\rangle^{1 / 2}} \exp \left(-\frac{t^{2}}{2\left\langle T_{c}^{2}\right\rangle}\right)
$$

where $\left\langle T_{c}^{2}\right\rangle$ is given by (17) in the general case and by (18) in case of a Gaussian pulse.

\section{PULSE WIDTH}

In terms of the Fourier components the pulse width $T_{w 2}$ reads:

$$
T_{w 2}^{2}=\frac{\int \hat{R}(\omega) \hat{E}_{0}(\omega) d \omega}{\int \hat{E}_{0}(\omega) d \omega}
$$

where

$$
\hat{R}(\omega):=\frac{\left|\hat{u}^{\prime}\right|^{2}(\omega)+\left|\hat{v}^{\prime}\right|^{2}(\omega)}{|\hat{u}|^{2}(\omega)+|\hat{v}|^{2}(\omega)} .
$$

The process $\hat{R}(\omega, z)$ obeys a stochastic differential equation that reads:

$$
d \hat{R}=\sigma\left(\hat{r}_{1} \circ d W^{1}+\hat{r}_{2} \circ d W^{2}+\hat{r}_{3} \circ d W^{3}\right)
$$

where the vector $\hat{\mathbf{r}}(\omega)$ :

$$
\begin{aligned}
& \hat{r}_{1}(\omega)=2 \operatorname{Im}\left(\hat{u}^{\prime} \hat{u}^{*}-\hat{v}^{\prime} \hat{v}^{*}\right) \\
& \hat{r}_{2}(\omega)=2 \operatorname{Im}\left(\hat{u}^{\prime} \hat{v}^{*}+\hat{v}^{\prime} \hat{u}^{*}\right) \\
& \hat{r}_{3}(\omega)=2 \operatorname{Re}\left(\hat{u}^{\prime} \hat{v}^{*}-\hat{v}^{\prime} \hat{u}^{*}\right)
\end{aligned}
$$

is solution of:

$$
\hat{\mathbf{r}}_{z}=2 \sigma \omega \dot{\mathbf{W}}(z) \times \hat{\mathbf{r}}+2 \sigma \dot{\mathbf{W}}(z)
$$

The vector $\hat{r}$ is the so-called PMD vector. Let us denote $\tau(\omega, z)=4(\hat{R}(\omega, z)-\hat{R}(\omega, 0))$. We have $|\hat{\mathbf{r}}(\omega)|^{2}=\tau(\omega, z)$ and $\tau$ is the so-called square Differential Group Delay (DGD). Furthermore $\tau$ is a diffusion process with infinitesimal generator:

$$
\mathcal{L}=8 \sigma^{2} \tau \frac{\partial^{2}}{\partial \tau^{2}}+12 \sigma^{2} \frac{\partial}{\partial \tau}
$$

which implies that $\tau(\omega, z)$ obeys a $\chi^{2}$ distribution with three degrees of freedom. In other words the PDF of $\tau(\omega, z)$ is:

$$
p(\tau)=\frac{\tau^{1 / 2}}{\sqrt{2 \pi}\left(4 \sigma^{2} z\right)^{3 / 2}} \exp \left(-\frac{\tau}{8 \sigma^{2} z}\right) \mathbf{1}_{\tau \geq 0}
$$

Note that the pulse width $T_{w 2}(z)$ is thus always larger than $T_{w 2}(0)$ whatever $z$ and whatever the realization of the fiber since $\tau(z, \omega) \geq 0$. Besides the statistical distribution of $\tau$ is independent of $\omega$. In particular its mean grows linearly with length:

$$
\langle\tau\rangle=12 \sigma^{2} z
$$

The correlation function of $\tau$ at two nearby frequencies is required for the forthcoming statements. It is found that the process $\left(\tau_{1}, \tau_{2}, C_{p}\right)$ where $\tau_{1}=\tau\left(\omega_{1}\right), \tau_{2}=\tau\left(\omega_{2}\right)$, and $C_{p}=\hat{\mathbf{r}}\left(\omega_{1}\right) \cdot \hat{\mathbf{r}}\left(\omega_{2}\right)$ is a diffusion process with infinitesimal generator: 


$$
\begin{aligned}
\mathcal{L}= & 8 \sigma^{2} \tau_{1} \frac{\partial^{2}}{\partial \tau_{1}^{2}}+12 \sigma^{2} \frac{\partial}{\partial \tau_{1}}+8 \sigma^{2} \tau_{2} \frac{\partial^{2}}{\partial \tau_{2}^{2}}+12 \sigma^{2} \frac{\partial}{\partial \tau_{2}} \\
& +16 \sigma^{2} C_{p} \frac{\partial^{2}}{\partial \tau_{1} \partial \tau_{2}}+\sigma^{2}\left(12-4 \Delta \omega^{2} C_{p}\right) \frac{\partial}{\partial C_{p}} \\
& +2 \sigma^{2}\left(\Delta \omega^{2}\left(\tau_{1} \tau_{2}-C_{p}^{2}\right)+\tau_{1}+\tau_{2}+2 C_{p}\right) \frac{\partial^{2}}{\partial C_{p}^{2}} \\
& +8 \sigma^{2}\left(C_{p}+\tau_{1}\right) \frac{\partial^{2}}{\partial C_{p} \partial \tau_{1}}+8 \sigma^{2}\left(C_{p}+\tau_{2}\right) \frac{\partial^{2}}{\partial C_{p} \partial \tau_{2}}
\end{aligned}
$$

where $\Delta \omega=\omega_{2}-\omega_{1}$. The complete expression of the generator represents a straightforward generalization of previously known results. For instance, it allows one to recover the expression of the expectation of the correlation degree between the PMD vectors at two nearby frequencies:

$$
\left\langle C_{p}\right\rangle=\frac{3}{\Delta \omega^{2}}\left(1-e^{-4 \sigma^{2} \Delta \omega^{2} z}\right)
$$

which was first derived in [5]. We can also compute the covariance of the square DGD at two nearby frequencies $\omega_{1}$ and $\omega_{2}$ :

$$
\operatorname{Cov}\left(\tau\left(\omega_{1}\right), \tau\left(\omega_{2}\right)\right)=\frac{12}{\Delta \omega^{4}}\left(4 \Delta \omega^{2} \sigma^{2} z-1+e^{-4 \Delta \omega^{2} \sigma^{2} z}\right)
$$

which is consistent with the formula derived in [6]. Further we can compute the expected value of any combination of $C_{p}, \tau_{1}$, and $\tau_{2}$. For instance, the second moment of the correlation degree of $C_{p}$ is found to be:

$$
\begin{array}{r}
\left\langle C_{p}^{2}\right\rangle=\frac{1}{24 \Delta \omega^{2}}\left(4-9 e^{-4 \Delta \omega^{2} \sigma^{2} z}+5 e^{-12 \Delta \omega^{2} \sigma^{2} z}\right. \\
\left.+24 \Delta \omega^{2} \sigma^{2} z+72 \Delta \omega^{4} \sigma^{4} z^{2}\right)
\end{array}
$$

Once the statistical distribution of $\tau$ is known it is easy to compute the mean and variance of $T_{w 2}^{2}$ :

$$
\left\langle T_{w 2}^{2}\right\rangle=T_{0}^{2}+3 \sigma^{2} z
$$

where $T_{0}$ is the initial pulse width. Note that the linear growth of the square pulse width is a universal feature that does not depend on the shape of the initial pulse. This remarkable property is involved by the independence of the expected value of $\tau$ with respect to $\omega$. However the variance of $T_{w 2}^{2}$ depends on the shape of the initial pulse:

$$
\operatorname{var}\left(T_{w 2}^{2}\right)=\frac{\iint \hat{E}_{0}\left(\omega_{1}\right) \hat{E}_{0}\left(\omega_{2}\right) \operatorname{Cov}\left(\tau\left(\omega_{1}\right), \tau\left(\omega_{2}\right)\right) d \omega_{1} d \omega_{2}}{16\left(\int \hat{E}_{0}(\omega) d \omega\right)^{2}}
$$

In case of a Gaussian pulse:

$$
\operatorname{var}\left(T_{w 2}^{2}\right)=T_{0}\left(T_{0}^{2}+4 \sigma^{2} z\right)^{3 / 2}-6 T_{0}^{2} \sigma^{2} z-T_{0}^{4}
$$

More generally the following identity is valid whatever the input pulse:

$$
\frac{d \operatorname{var}\left(T_{w 2}^{2}\right)}{d z}=12 \sigma^{2}\left\langle T_{c}^{2}\right\rangle
$$

We can then deduce some properties of the variance:

$$
\begin{aligned}
\operatorname{var}\left(T_{w 2}^{2}\right) \stackrel{\sigma^{2} z}{ } \stackrel{\ll T_{0}^{2}}{\simeq} 6 \sigma^{4} z^{2} \\
\sigma^{2} z \gg T_{0}^{2} 8 \sqrt{\pi} c_{1}\left(\sigma^{2} z\right)^{3 / 2}
\end{aligned}
$$

Let us now compute the PDF of $T_{w 2}^{2}$ :

$$
\int_{t^{2}}^{\infty} p(u) d u=\mathbb{P}\left(T_{w 2}^{2} \geq t^{2}\right)
$$

It can be readily estimated in the asymptotic configurations $\sigma^{2} z \ll T_{0}$ or $\gg T_{0}^{2}$. If $\sigma^{2} z \ll T_{0}^{2}$ then the processes $\tau(\omega)$ are completely correlated for $\omega$ in the spectrum of the pulse so that the distribution of $T_{w 2}^{2}$ is:

$$
p\left(t^{2}\right) \stackrel{\sigma^{2} z \ll T_{0}^{2}}{=} \frac{\sqrt{t^{2}-T_{0}^{2}}}{\sqrt{2 \pi}\left(\sigma^{2} z\right)^{3 / 2}} \exp \left(-\frac{t^{2}-T_{0}^{2}}{2 \sigma^{2} z}\right) .
$$

If $\sigma^{2} z \gg T_{0}^{2}$ then the processes $\tau$ are uncorrelated with respect to $\omega$ by Eq. (19), so that $T_{w 2}^{2}$ is the sum of large number of uncorrelated components. The central limit theorem can then be invoked to establish that the statistics of $T_{w 2}^{2}$ is Gaussian. Since the mean value and variance of $T_{w 2}^{2}$ are known, this implies that the PDF of $T_{w 2}^{2}$ is:

$$
p\left(t^{2}\right) \stackrel{\sigma^{2} z \gg T_{0}^{2}}{=} \frac{1}{\sqrt{2 \pi \operatorname{var}\left(T_{w 2}^{2}\right)}} \exp \left(-\frac{\left(t^{2}-\left\langle T_{w 2}^{2}\right\rangle\right)^{2}}{2 \operatorname{var}\left(T_{w 2}^{2}\right)}\right) .
$$

Note that the decorrelation rate between $\tau\left(\omega_{1}\right)$ and $\tau\left(\omega_{2}\right)$ is very slow (as $1 / \Delta \omega^{2}$ ), so that the second regime can be observed only when $\sigma^{2} z$ is much larger than $T_{0}^{2}$. Besides the same argument shows that the first expression of the PDF should be valid even for $\sigma^{2} z \sim T_{0}^{2}$, especially for the probability tail $p\left(t^{2}\right), t^{2} \gg T_{0}^{2}$, if we take care to take into account the exact expression of the variance of $T_{w 2}^{2}$ :

$$
p\left(t^{2}\right) \stackrel{\sigma^{2} z \leq T_{0}^{2}}{\simeq} \frac{\sqrt{t^{2}-T_{2}^{2}}}{\sqrt{2 \pi} \gamma_{2}^{3 / 2}} \exp \left(-\frac{t^{2}-T_{2}^{2}}{2 \gamma_{2}}\right)
$$

where

$$
\begin{aligned}
\gamma_{2} & =\left(\operatorname{var}\left(T_{w 2}^{2}\right) / 6\right)^{1 / 2} \\
T_{2}^{2} & =\left\langle T_{w 2}^{2}\right\rangle-\left(3 \operatorname{var}\left(T_{w 2}^{2}\right) / 2\right)^{1 / 2}
\end{aligned}
$$

If we consider $T_{w 1}$ the results read as follows. The mean value of $T_{w 1}^{2}$ is simply the difference between the mean values of $T_{w 2}^{2}$ and $T_{c}^{2}$. The variance of $T_{w 1}^{2}$ is:

$$
\operatorname{var}\left(T_{w 1}^{2}\right)=\operatorname{var}\left(T_{w 2}^{2}\right)+\operatorname{var}\left(T_{c}^{2}\right)-2 \operatorname{Cov}\left(T_{w 2}^{2}, T_{c}^{2}\right)
$$

Calculations show that

$$
\operatorname{var}\left(T_{w 1}^{2}\right)=\operatorname{var}\left(T_{w 2}^{2}\right)-2\left\langle T_{c}^{2}\right\rangle^{2}
$$


In case of a Gaussian pulse we get:

$$
\left\langle T_{w 1}^{2}\right\rangle=T_{0}^{2}+3 \sigma^{2} z-\frac{T_{0}^{2}}{2}\left(\sqrt{1+\frac{4 \sigma^{2} z}{T_{0}^{2}}}-1\right)
$$

Note that this expression was also derived in Ref. [5] The variance of $T_{w 1}^{2}$ is:

$$
\operatorname{var}\left(T_{w 1}^{2}\right)=T_{0}^{4} \sqrt{1+\frac{4 \sigma^{2} z}{T_{0}^{2}}}\left(\sqrt{1+\frac{4 \sigma^{2} z}{T_{0}^{2}}}-1\right)^{2}
$$

For a general pulse shape, the mean value of $T_{w 1}^{2}$ grows linearly:

$$
\begin{aligned}
& \left\langle T_{w 1}^{2}\right\rangle \stackrel{\sigma^{2} z \ll T_{0}^{2}}{\simeq} T_{0}^{2}+2 \sigma^{2} z \\
& \sigma^{2} z \gg T_{0}^{2} 3 \sigma^{2} z
\end{aligned}
$$

while the variance satisfies:

$$
\begin{aligned}
\operatorname{var}\left(T_{w 1}^{2}\right) \stackrel{\sigma^{2} z}{\simeq} & \ll T_{0}^{2} \\
\sigma^{2} z & \gg \sigma_{0}^{2} z^{2} \\
& \simeq \sqrt{\pi} c_{1}\left(\sigma^{2} z\right)^{3 / 2}
\end{aligned}
$$

Let us now estimate the PDF of $T_{w 1}^{2}$. If $\sigma^{2} z \ll T_{0}^{2}$ then we know that $T_{w 1}^{2}=T_{0}^{2}+\sigma^{2} W_{z}^{2}+\sigma^{2} W_{z}^{3}+O\left(\sigma^{4} z^{2} / T_{0}^{4}\right)$ so that it obeys a $\chi^{2}$ distribution with two degrees of freedom:

$$
p\left(t^{2}\right) \stackrel{\sigma^{2} z \ll T_{0}^{2}}{=} \frac{1}{\sigma^{2} z} \exp \left(-\frac{t^{2}-T_{0}^{2}}{2 \sigma^{2} z}\right), \quad t^{2}>T_{0}^{2} .
$$

If $\sigma^{2} z \gg T_{0}^{2}$ then $T_{w 2}^{2}$ is the prevailing term in the expression of $T_{w 1}^{2}$ so that their PDF are common. In the general configuration, $T_{w 2}^{2}$ obeys a $\chi^{2}$ distribution with three degrees of freedom, while $T_{c}^{2}$ is the square of a Gaussian random variable, so that we can guess that the PDF of $T_{w 1}^{2}$ corresponds to a $\chi^{2}$ distribution with a number $d_{1}$ of degrees of freedom which is between 2 and 3 . Since we know the mean and variance of $T_{w 1}^{2}$ we can fix the free parameters:

$$
d_{1}=\left(\left\langle T_{w 1}^{2}\right\rangle-T_{0}^{2}\right) /\left(\sigma^{2} z\right)
$$

so that:

$$
p\left(t^{2}\right)=\frac{\left(t^{2}-T_{1}^{2}\right)^{d_{1} / 2-1}}{2^{d_{1} / 2} \Gamma\left(d_{1} / 2\right) \gamma_{1}^{d_{1} / 2}} \exp \left(-\frac{t^{2}-T_{1}^{2}}{2 \gamma_{1}}\right)
$$

where $\Gamma(s)=\int_{0}^{\infty} t^{s-1} e^{-t} d t$ and

$$
\begin{aligned}
\gamma_{1} & =\left(\operatorname{var}\left(T_{w 1}^{2}\right) /\left(2 d_{1}\right)\right)^{1 / 2} \\
T_{1}^{2} & =\left\langle T_{w 1}^{2}\right\rangle-\left(d_{1} \operatorname{var}\left(T_{w 1}^{2}\right) / 2\right)^{1 / 2}
\end{aligned}
$$

\section{ROTATION OF POLARIZATION}

The rotation of the pulse polarization can be characterized by the ratio $P_{r}$ of the energy on the input polarization state over the total energy. In terms of the first Stokes parameter the parameter $P_{r}$ reads as:

$$
P_{r}=\frac{1}{2}+\frac{1}{2} \frac{\int \hat{s}_{1}(\omega) d \omega}{\int \hat{E}_{0}(\omega) d \omega}
$$

The computation of the mean and covariance of $\hat{s}_{1}$ is straightforward:

$$
\begin{gathered}
\left\langle\hat{s}_{1}(\omega)\right\rangle=\hat{E}_{0}(\omega) \exp \left(-4 \omega^{2} \sigma^{2} z\right) \\
\operatorname{Cov}\left(\hat{s}_{1}\left(\omega_{1}\right), \hat{s}_{1}\left(\omega_{2}\right)\right)=\hat{E}_{0}\left(\omega_{1}\right) \hat{E}_{0}\left(\omega_{2}\right)\left(\frac{1}{3} e^{-4\left(\omega_{1}-\omega_{2}\right)^{2} \sigma^{2} z}\right. \\
\left.+\frac{2}{3} e^{-4\left(\omega_{1}^{2}+\omega_{2}^{2}+\omega_{1} \omega_{2}\right) \sigma^{2} z}-e^{-4\left(\omega_{1}^{2}+\omega_{2}^{2}\right) \sigma^{2} z}\right)
\end{gathered}
$$

We can then state that the mean of $P_{r}$ is:

$$
\left\langle P_{r}\right\rangle=\frac{1}{2}+\frac{1}{2} \frac{\int \exp \left(-4 \omega^{2} \sigma^{2} z\right) \hat{E}_{0}(\omega) d \omega}{\int \hat{E}_{0}(\omega) d \omega}
$$

which is equal for a Gaussian pulse to:

$$
\left\langle P_{r}\right\rangle=\frac{1}{2}+\frac{1}{2}\left(1+\frac{2 \sigma^{2} z}{T_{0}^{2}}\right)^{-1 / 2}
$$

For a general pulse, we have:

$$
\begin{aligned}
\left\langle P_{r}\right\rangle \stackrel{\sigma^{2} z}{ } \stackrel{\ll T_{0}^{2}}{\simeq} 1-2 \sigma^{2} z c_{2} \\
\sigma^{2} z \gg T_{0}^{2} \frac{1}{2}+\frac{c_{3} \sqrt{\pi}}{4 \sqrt{\sigma^{2} z}}
\end{aligned}
$$

where $c_{3}=\frac{\hat{E}_{0}(0)}{\int \hat{E}_{0}(\omega) d \omega}=\frac{1}{2 \pi} \frac{\left|\int u_{0} d t\right|^{2}}{\int\left|u_{0}\right|^{2} d t}$.

The fluctuations around the mean value can be estimated by the computation of the variance of $P_{r}$ :

$$
\operatorname{var}\left(P_{r}\right)=\frac{1}{4} \frac{\iint \operatorname{Cov}\left(\hat{s}_{1}\left(\omega_{1}\right), \hat{s}_{1}\left(\omega_{2}\right)\right) d \omega_{1} d \omega_{2}}{\left(\int \hat{E}_{0}(\omega) d \omega\right)^{2}}
$$

For a Gaussian pulse:

$$
\begin{aligned}
\operatorname{var}\left(P_{r}\right)=\frac{1}{12} & \left(1+\frac{4 \sigma^{2} z}{T_{0}^{2}}\right)^{-1 / 2}-\frac{1}{4}\left(1+\frac{2 \sigma^{2} z}{T_{0}^{2}}\right)^{-1} \\
& +\frac{1}{6}\left(1+\frac{3 \sigma^{2} z}{T_{0}^{2}}\right)^{-1 / 2}\left(1+\frac{\sigma^{2} z}{T_{0}^{2}}\right)^{-1 / 2}
\end{aligned}
$$

For a general pulse, we have:

$$
\begin{aligned}
\operatorname{var}\left(P_{r}\right) \stackrel{\sigma^{2} z \ll T_{0}^{2}}{\simeq} 16 \sigma^{4} z^{2} c_{2}^{2} \\
\stackrel{\simeq}{\sigma^{2} z T_{0}^{2}} \frac{\sqrt{\pi} c_{1}}{6 \sqrt{\sigma^{2} z}}
\end{aligned}
$$




\section{DEGREE OF POLARIZATION}

The degree of polarization $P_{d}$ can be expressed in terms of the frequency correlation degree of the Stokes vectors as:

$$
P_{d}=\frac{\sqrt{\iint C\left(\omega_{1}, \omega_{2}\right) \hat{E}_{0}\left(\omega_{1}\right) \hat{E}_{0}\left(\omega_{2}\right) d \omega_{1} d \omega_{2}}}{\int \hat{E}_{0}(\omega) d \omega}
$$

It is then easy to compute the mean of $P_{d}^{2}$. One then gets that the degree of polarization and the time displacement satisfy the equation:

$$
\frac{d\left\langle T_{c}^{2}\right\rangle}{d z}=\sigma^{2}\left\langle P_{d}^{2}\right\rangle
$$

For a Gaussian pulse we have:

$$
\left\langle P_{d}^{2}\right\rangle=\left(1+\frac{4 \sigma^{2} z}{T_{0}^{2}}\right)^{-1 / 2}
$$

For a general pulse the degree of polarization first decays linearly:

$$
\left\langle P_{d}^{2}\right\rangle \stackrel{\sigma^{2} z \ll T_{0}^{2}}{\simeq} 1-8 \sigma^{2} z\left(c_{2}-c_{4}^{2}\right)
$$

while it slowly decays to 0 for long propagation distances:

$$
\left\langle P_{d}^{2}\right\rangle \stackrel{\sigma^{2} z \gg T_{0}^{2}}{\simeq} \frac{\sqrt{\pi} c_{1}}{2 \sigma \sqrt{z}}
$$

More exactly, if $\sigma^{2} z \ll T_{0}^{2}$, then the PDF of the random variable $1-P_{d}$ is:

$$
p(x)=\frac{1}{4 \sigma^{2}\left(c_{2}-c_{4}^{2}\right) z} \exp \left(-\frac{x}{4 \sigma^{2}\left(c_{2}-c_{4}^{2}\right) z}\right) .
$$

If $\sigma^{2} z \gg T_{0}^{2}$, then the PDF of $P_{d}$ is:

$$
p(x)=\frac{\sqrt{2}}{\sqrt{\pi} \alpha^{3 / 2}} x^{2} \exp \left(-\frac{x^{2}}{2 \alpha}\right)
$$

where $\alpha(z)=\left(\sqrt{\pi} c_{1}\right) /(6 \sigma \sqrt{z})$.

\section{INFLUENCE OF GVD}

If we take into account both GVD and PMD, then the field $\mathbf{U}=(u, v)^{T}$ satisfies Eq. (2). In terms of the Fourier components $\hat{\mathbf{U}}=(\hat{u}, \hat{v})^{T}$ this partial differential equation reads as a collection of ordinary differential equations:

$$
\hat{\mathbf{U}}_{z}=i \omega R(z) \hat{\mathbf{U}}+i \frac{\beta^{\prime \prime}}{2} \omega^{2} \hat{\mathbf{U}}
$$

Setting $\check{\mathbf{U}}=\hat{\mathbf{U}} \exp \left(-i \beta^{\prime \prime} \omega^{2} z / 2\right)$ the system of differential equations for $\check{\mathbf{U}}$ is the same as in the absence of
GVD. The relevant quantities $\hat{E}_{0}(\omega), \hat{R}(\omega)$, and $\hat{t}(\omega)$ can be expressed as:

$$
\begin{aligned}
\hat{E}_{0}(\omega) & =\check{E}_{0}(\omega) \\
\hat{R}(\omega) & =\check{R}(\omega)+\beta^{\prime \prime 2} \omega^{2} z^{2} \check{E}_{0}(\omega)+2 \beta^{\prime \prime} \omega z \check{I}(\omega) \\
\hat{t}(\omega) & =\check{t}(\omega)+\beta^{\prime \prime} \omega z \check{E}_{0}(\omega)
\end{aligned}
$$

where $\check{E}_{0}, \check{R}$, and $\check{t}$ are defined in terms of $\check{u}$ and $\check{v}$ similarly as $\hat{E}_{0}, \hat{R}$, and $\hat{t}$. We know from the previous sections the statistical distributions of $\check{E}_{0}, \check{R}$, and $\check{t}$, so that it is easy to get the statistical distributions of $\hat{E}_{0}, \hat{R}$, and $\hat{t}$.

Let us first address the timing displacement. Only the mean value of the time displacement is modified by the presence of GVD:

$$
\begin{aligned}
\left\langle T_{c}\right\rangle & =\beta^{\prime \prime} c_{4} z \\
\operatorname{var}\left(T_{c}\right) & =\left.\operatorname{var}\left(T_{c}\right)\right|_{\beta^{\prime \prime}=0}
\end{aligned}
$$

For the Gaussian pulse (12) we have $c_{4}=0$, which shows that GVD has no influence on the timing displacement. Let us now consider the pulse broadening. Both the mean value and variance of the pulse width are enhanced by the GVD:

$$
\begin{aligned}
&\left\langle T_{w 2}^{2}\right\rangle=T_{0}^{2}+3 \sigma^{2} z+c_{2} \beta^{\prime \prime 2} z^{2} \\
& \operatorname{var}\left(T_{w 2}^{2}\right)=\left.\operatorname{var}\left(T_{w 2}^{2}\right)\right|_{\beta^{\prime \prime}=0} \\
&+4 \beta^{\prime \prime}{ }^{2} z^{2} \frac{\iint \omega_{1} \omega_{2}\left\langle\check{t}\left(\omega_{1}\right) \check{t}\left(\omega_{2}\right)\right\rangle \check{E}_{0}\left(\omega_{1}\right) \check{E}_{0}\left(\omega_{2}\right) d \omega_{1} d \omega_{2}}{\left(\int \check{E}_{0}(\omega) d \omega\right)^{2}} \\
&\left\langle T_{w 1}^{2}\right\rangle=T_{0}^{2}+3 \sigma^{2} z+\left(c_{2}+c_{4}^{2}\right) \beta^{\prime \prime 2} z^{2}+\left.\operatorname{var}\left(T_{c}\right)\right|_{\beta^{\prime \prime}=0} \\
& \operatorname{var}\left(T_{w 1}^{2}\right)=\operatorname{var}\left(T_{w 2}^{2}\right)-2 \operatorname{var}\left(T_{c}\right)^{2}
\end{aligned}
$$

For the Gaussian pulse we have:

$$
\begin{aligned}
\left\langle T_{w 2}^{2}\right\rangle= & T_{0}^{2}+3 \sigma^{2} z+\frac{\beta^{\prime \prime 2} z^{2}}{4 T_{0}^{2}} \\
\operatorname{var}\left(T_{w 2}^{2}\right)= & T_{0}^{4}\left(\left(1+\frac{4 \sigma^{2} z}{T_{0}^{2}}\right)^{3 / 2}-6 \frac{\sigma^{2} z}{T_{0}^{2}}-1\right) \\
& +\frac{\beta^{\prime \prime} z^{2}}{4} \frac{\left(\sqrt{1+\frac{4 \sigma^{2} z}{T_{0}^{2}}}-1\right)^{2}}{\sqrt{1+\frac{4 \sigma^{2} z}{T_{0}^{2}}}}
\end{aligned}
$$

$$
\begin{aligned}
\left\langle T_{w 1}^{2}\right\rangle= & T_{0}^{2}+3 \sigma^{2} z+\frac{\beta^{\prime \prime 2} z^{2}}{4 T_{0}^{2}}-\frac{T_{0}^{2}}{2}\left(\sqrt{1+\frac{4 \sigma^{2} z}{T_{0}^{2}}}-1\right) \\
\operatorname{var}\left(T_{w 1}^{2}\right)= & T_{0}^{4} \sqrt{1+\frac{4 \sigma^{2} z}{T_{0}^{2}}}\left(\sqrt{1+\frac{4 \sigma^{2} z}{T_{0}^{2}}}-1\right)^{2} \\
& \times\left(1+\frac{\beta^{\prime \prime 2} z^{2}}{4 T_{0}^{4}\left(1+\frac{4 \sigma^{2} z}{T_{0}^{2}}\right)}\right)
\end{aligned}
$$

The degree of polarization is not affected by the presence of GVD. 


\section{NUMERICAL SIMULATIONS}

Here we solve numerically the propagation equation in absence of GVD. We consider a Gaussian pulse or a sech pulse as an initial condition with an initial rms width $T_{0}=4 \mathrm{ps}$. We assume that the magnitude of linear birefringence is constant. In order to simulate the random fluctuations of birefringence we incorporate at fictitious junctions between adjacent fiber pieces with length $\Delta z$ random axial rotation and addition of random phase difference between the field components. We take $\Delta \beta^{\prime}=2$ $\mathrm{ps} / \mathrm{km}$, and $\Delta z=25 \mathrm{~m}$ so that $\sigma^{2}=8.310^{-3} \mathrm{ps}^{2} / \mathrm{km}$. Note that this corresponds to a configuration where the usual parameter $D_{p}=0.29 \mathrm{ps} / \sqrt{\mathrm{km}}$. The number of samples used to evaluate averaged values and variances are $10^{4}$. Comparison of the numerical results and theoretical formulas show excellent agreement (Figures 2-3).

(a)

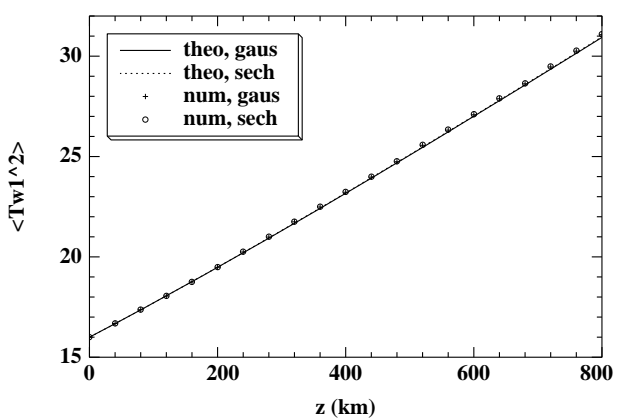

(b)

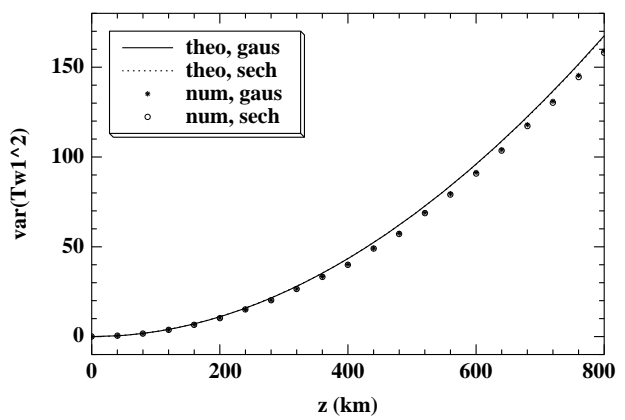

FIG. 2. Square rms widths of pulses with gauss or sech shapes. The lines correspond to the theoretical values while the crosses and circles represent the results averaged over $10^{4}$ numerical simulations. The mean values are plotted in picture $a$, the variances are plotted in picture $b$.

(a)

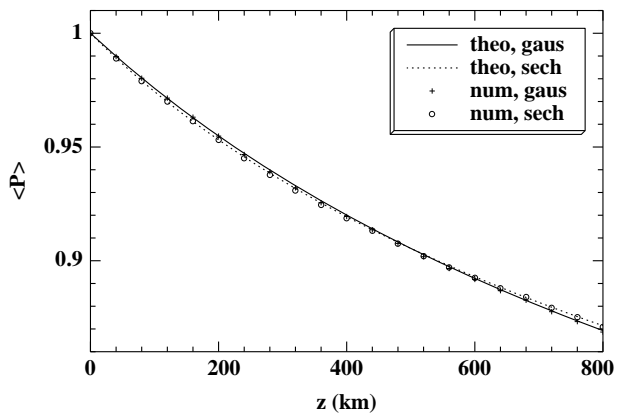

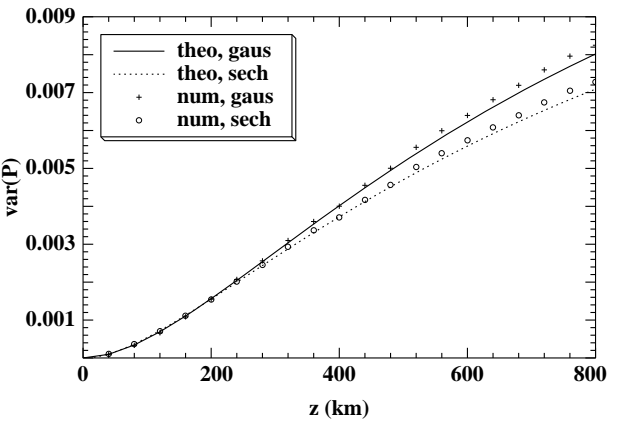

FIG. 3. Rotations of the polarization of pulses with gauss or sech shapes. The lines correspond to the theoretical values while the crosses and circles represent the results averaged over $10^{4}$ numerical simulations. The mean values are plotted in picture $a$, the variances are plotted in picture $b$.

Another series of runs have been carried out where the local birefringence strength and angle vary with distance. We present in Figures 4-5 the results corresponding to two configurations where $\sigma^{2} z / T_{0}^{2}=0.12(\ll 1)$ and $\sigma^{2} z / T_{0}^{2}=2.2$ respectively. The agreements with the theoretical formulas are still excellent. In particular the numerical histograms of the pulse widths are found very close to the theoretical PDF.

(a)
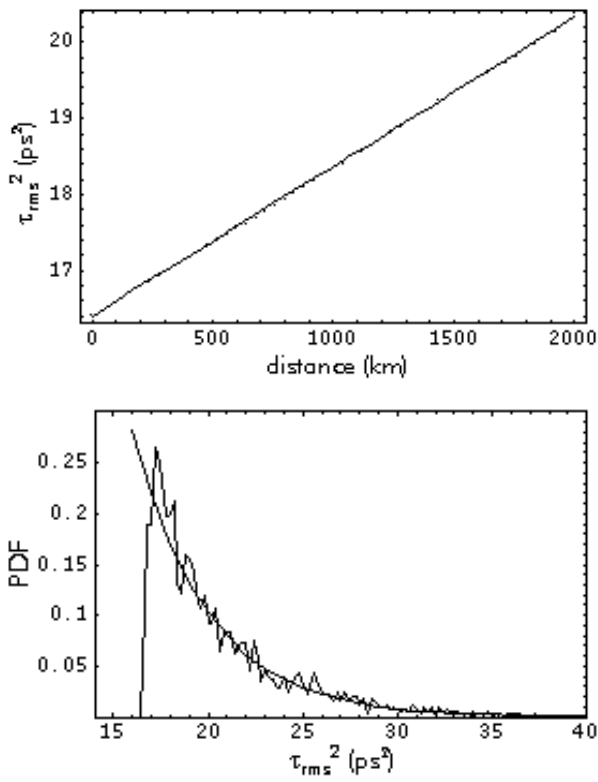

(b)

FIG. 4. Mean square width (picture a) and PDF of the pulse width (picture b) of pulses with gauss shapes. The numerical values have been computed from 3000 runs. The PDF is plotted at $z=2000 \mathrm{~km}$. Here $T_{0}^{2}=16.4 \mathrm{ps}^{2}$ and $D_{p}=0.1$ $\mathrm{ps} / \sqrt{\mathrm{km}}$ The PDF is plotted at $z=2000 \mathrm{~km}$ which corresponds to $\sigma^{2} z / T_{0}^{2}=0.12$. The theoretical PDF is formula (27). 
(a)

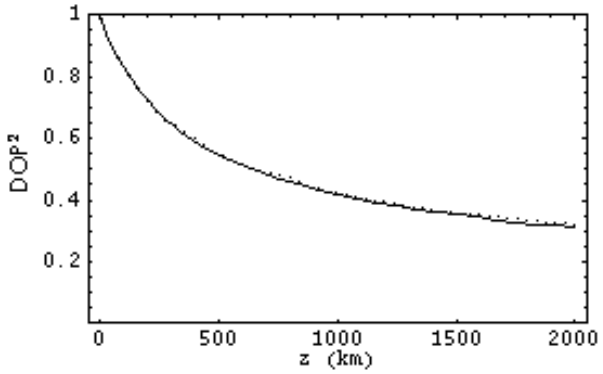

(b)

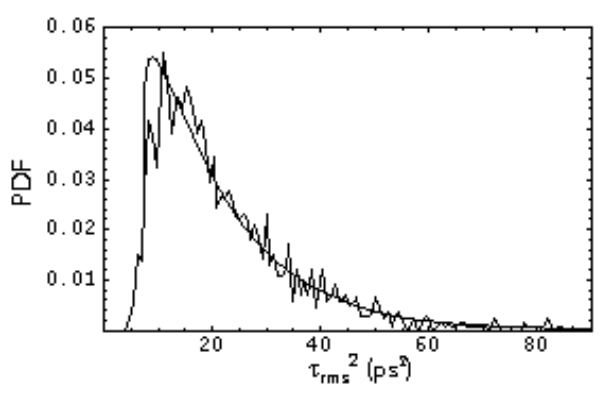

FIG. 5. Degree of polarization (picture a) and PDF of the pulse width (picture b) of pulses with gauss shapes. The numerical values have been computed from 3000 runs. Here $T_{0}^{2}=3.6 \mathrm{ps}^{2}$ and $D_{p}=0.2 \mathrm{ps} / \sqrt{\mathrm{km}}$. The PDF is plotted at $z=2000 \mathrm{~km}$ which corresponds to $\sigma^{2} z / T_{0}^{2}=2.2$. The theoretical PDF is formula (28).

\section{CONCLUSION}

In this paper we have studied behaviors of short pulses in fibers with randomly varying birefringence. We have derived closed form expressions for the statistical values of relevant quantities. The mean values, variances and PDF of the time displacement, time rms width and degree of polarization have been computed. This gives further insight in the PMD dynamic, as well as practical help for engineers in that not only the mean values averaged over an ensemble of fibers are given. As remarked in [6], the statistical properties of PMD in long fibers are uniquely determined by the mean DGD. It could be also interesting to take into account polarization-dependent losses. This could certainly be done, as the statistical distribution of the DGD was carried out in [19].

* The present address of J. Fatome is: Laboratoire de Physique de l'Université de Bourgogne, Avenue Alain Savary, 21000 Dijon, France

\section{REFERENCES}

[1] E. Kollveit, P. A. Andrekson, J. Brentel, B. E. Olsson, B. Bahkshi, J. Handryd, P. O. Hedekvist, M. Karlsson, H. Sunnerud, and J. Li, Electron. Lett. 35 (1999) 75.

[2] G. J. Foschini and C. D. Poole, J. Lightwave Technol. 9 (1991) 1439.
[3] C. R. Menyuk and P. K. A. Wai, J. Opt. Soc. Am. B 11, 1288 (1994).

[4] P. K. A. Wai and C. R. Menyuk, J. Lightwave Technol. 14, 148 (1996).

[5] M. Karlsson and J. Brentel, Opt. Lett. 24, 939 (1999).

[6] M. Shtaif and A. Mecozzi, Opt. Lett. 25 (2000) 707.

[7] F. Bruyere, Opt. Fiber Technol. 2 (1996) 269.

[8] C. R. Menyuk, IEEE J. Quantum Electron. 25, 2674 (1989).

[9] M. Matsumoto, Y. Akagi, and A. Hasegawa, J. Lightwave Technol. 15, 584 (1997).

[10] C. D. Poole and R. E. Wagner, Electron. Lett. 22, 1029 (1986); C. D. Poole and D. L. Favin, J. Lightwave Technol. 12, 917 (1994).

[11] M. C. de Lignie, H. G. J. Nagel, and M. O. van Deventer, J. Lightwave Technol. 12, 1325 (1994).

[12] A. Galtarossa, G. Gianello, C. G. Someda, and M. Schiano, J. Lightwave Technol. 14, 42 (1996).

[13] N. Gisin and J. P. Pellaux, Opt. Commun. 89 (1992) (1992).

[14] T .I. Lakoba and D. J. Kaup, Phys. Rev. E 56, 6147 (1997).

[15] C. Xie, M. Karlsson, and P. A. Andrekson, IEEE Photon. Technol. Lett. 12, 801 (2000).

[16] M. Karlsson, Opt. Lett. 23 (1998) 688.

[17] M. Born and E. Wolf, Principles of optics (Pergamon, Oxford, 1980).

[18] M. Abramowitz and I. Stegun, Handbook of mathematical functions (Dover Publications, New-York, 1965).

[19] Y. Li and A. Yariv, J. Opt. Soc. Am. B 17 (2000) 1821. 